\title{
¿Cuáles son las variables clave en la adopción del marketing de relaciones? Una investigación en el contexto colombiano
}

What are the Key Variables in the Adoption of Relationship Marketing? A Research in the Colombian Context

Quais são as variáveis chave na adoção do marketing de relações? Uma pesquisa no contexto colombiano

\section{César Augusto Salazar}

Fecha de recibido: 22 de octubre de 2013

Fecha de aprobado: 7 de mayo de 2014

Doi: dx.doi.org/10.12804/rev.univ.empresa.27.2014.02

Para citar este artículo: Salazar, C. A. (2014). ¿Cuáles son las variables clave en la adopción del marketing de relaciones? Una investigación en el contexto colombiano. Universidad \& Empresa, 16(27), 63-79. doi: dx. doi. org/10.12804/rev.univ.empresa.27.2014.02

\section{RESUMEN}

El objetivo de esta investigación es descubrir cuáles son las variables clave en la aplicación del marketing de relaciones en las empresas colombianas. Se aplica un instrumento comparativo a compañías pertenecientes a diferentes sectores y con distintos tamaños, en ese país, en dos olas: el primero, denominado 'ola 1', corresponde a los años 2003 y

Doctorado en Nuevas Tendencias en Dirección de Empresas de la Universidad de León España. Profesor de posgrado en múltiples Universidades en Colombia (Externado de Colombia, CESA, Sabana, entre otras) y profesor invitado en la Universidad de León (España). Gerente General de Thinking Marketing Intelligence -TESI Colombia, para la región Andina de Naciones. Correo electrónico: cesar.salazar@tmiandino.com 
2004; el segundo, denominado 'ola 2', corresponde a los años 2010 y 2011. La técnica utilizada para validar el constructo propuesto fue el análisis factorial de componentes principales.

Palabras clave: estrategias de marketing, marketing, marketing de relaciones, variables de marketing.

\section{ABSTRACT}

The purpose of this research is to discover the key variables in the application of relationship marketing in Colombian companies. A comparative instrument is applied to companies belonging to different industries and of different sizes in that country, in two waves or periods: the first, referred to as "wave 1", covers the years 2003 and 2004; the second, called "wave 2", covers the years 2010 and 2011. The technique used to validate the construct proposed was the factorial analysis of the principal components.

Keywords: Marketing, marketing strategies, marketing variables, relationship marketing.

\section{RESUMO}

O objetivo desta pesquisa é descobrir quais são as variáveis chave na aplicação do marketing de relações nas empresas colombianas. Aplica-se um instrumento comparativo a empresas pertencentes a diferentes setores e com diferentes tamanhos, nesse país, em duas etapas: a primeira denominada "ola l" corresponde aos anos 2003 e 2004; a segunda, denominada "ola 2", corresponde aos anos 2010 e 2011 . A técnica utilizada para validar o constructo proposto foi a análise fatorial de componentes principais.

Palavras-chave: estratégias de marketing, marketing, marketing de relações, variáveis de marketing. 


\section{INTRODUCCIÓN}

El marketing de relaciones tomó gran importancia en el mundo académico a partir de la segunda mitad de la década del ochenta, con contribuciones y aportaciones importantes provenientes de la escuela nórdicaeuropea, en la que se destacan autores como Christian Grönroos y Evert Gummesson.

Esta investigación se realizó con el fin de establecer la esencia del marketing de relaciones, de evaluar si las empresas - considerando para ello las colombianas - entienden este concepto y de determinar qué variables aplican dentro de la gestión de marketing que ellas realizan. Con estos propósitos en mente se diseñó, a partir de una revisión exhaustiva de las propuestas teóricas que sobre el tema han sido planteadas desde la década del ochenta hasta la fecha, un instrumento que recoge las principales variables y constructos de interés.

Antes de iniciar el análisis de componentes principales se decidió validar la existencia de correlación en las variables a través del modelo de correlación de Spearman. Se estableció la existencia de correlaciones bivariadas que son estadísticamente significativas $(p<0.01)$. Esto permite concluir que al cumplirse las relaciones entre las variables se hace pertinente la ejecución del análisis de componentes principales con el propósito de reducir el número de variables originales a un grupo menor de factores que faciliten establecer las relaciones subyacentes de las variables originales.

Elobjetivo del análisis de componentes principales fue el de reducir las variables del instrumento y aprovechar las puntuaciones factoriales de los componentes retenidos, para tratarlas como nuevas variables (Hair, Anderson, Tatham, \& Black, 1999).

\section{MARCO TEÓRICO}

Después de revisar los aspectos más relevantes de las definiciones provistas por Ballantyne (1999), Berry (2002), El-Ansary (2005), Grönroos (1990), Gummesson (1994) y Siems (2010), es posible ofrecer la siguiente definición sintética de marketing de relaciones:

Es un proceso continuo de comprometerse en actividades y programas de colaboración con consumidores inmediatos, usuarios finales, proveedores, empleados y todos los actores que intervienen en la relación para crear, mantener y mejorar mutuo valor económico.

Harker (1999), en su intento por alcanzar una definición, concluye que 
existen siete categorías conceptuales fundamentales con las que es posible definir el marketing de relaciones, a saber: nacimiento, desarrollo, mantenimiento, escala de tiempo, interacción, resultados y contenido emocional. Afirma, con base en una investigación de 117 fuentes distintas, que la mejor definición ofrecida y coincidente con estas categorías es la expuesta por Grönroos (1994). Esta definición plantea que el

\section{Relationship Marketing consiste en identificar y establecer, mantener e incrementar $y$, cuando es necesario, terminar las relaciones con clientes y otros actores en esta, en un am- biente provechoso, donde los obje- tivos de las partes se reúnen, lo cual se logra mediante el intercambio y cumplimiento de promesas (p. 9).}

A partir de esta definición se puede establecer que el tratamiento de las promesas en el marketing de relaciones permite lograr el cumplimiento de los objetivos de larelación. Bitner (1995) intenta categorizar el tratamiento de promesas en el contexto de la relación así: hacer, habilitar y mantener las promesas. El marketing de relaciones se mide mediante la construcción de confianza, metas, beneficios compartidos y trata- miento armonioso en las diferencias y desacuerdos (Abramson \& Ai, 1998). Morgan y Hunt (1994), por su parte, proponen que el centro del marketing de relaciones es la generación de compromiso y confianza entre las partes involucradas en la relación.

El marketing de relaciones involucra algo más que los clientes finales y su conocimiento Aeron, Kumar y Moorthy (2012), así como la generación de valor. Gracias a él se logra construir relaciones con otros actores que inciden en la participación de la empresa en un entorno ampliado. De hecho, para autores como Grönroos (2000) y Ballantyne (1999), la satisfacción del cliente, que se logra a través de él, genera lealtad y, por consiguiente, el establecimiento de una relación perdurable.

En concordancia con la literatura revisada a lo largo de este estudio, se establecen dos grandes colecciones de variables que, observadas al interior de cada grupo considerado en su interacción, permiten determinar las variables clave de adopción del marketing de relaciones en las empresas, independientemente de su tamaño (tabla 1). 
Tabla 1. Constructos aplicados en el instrumento

\begin{tabular}{l|l}
\hline \multicolumn{1}{c|}{ Constructo } & \multicolumn{1}{c}{ Fuente } \\
\hline $\begin{array}{l}\text { Áreas de la empresa que inciden en la toma de } \\
\text { decisiones (10 ítems) }\end{array}$ & Elaboración propia \\
\hline Método de recolectar información del mercado & Elaboración propia \\
\hline $\begin{array}{l}\text { Determinación de la orientación de la empresa } \\
\text { hacia las necesidades del cliente }\end{array}$ & $\begin{array}{l}\text { Ali, Jolson y Darmon (2004), Linvol y Razzouk } \\
\text { (1995), Sin et al. (2005), Snow (1997), Storbacka } \\
\text { (2000), Tadepalli y Ávila (1999) y Wrenn (1997) }\end{array}$ \\
\hline $\begin{array}{l}\text { Nivel de adopción del marketing de relaciones } \\
\text { en la gestión de marketing de la empresa (im- } \\
\text { portancia y desempeño). (19 ítems cada uno) }\end{array}$ & $\begin{array}{l}\text { Berry (1995), Bowen y Chen (2001); Butcher, } \\
\text { (1994), Grönroos (1994), Kavali, Tzokas y Saren } \\
\text { (1999) y Morgan y Hunt (1994) }\end{array}$ \\
\hline \begin{tabular}{l} 
Base de datos y su desempeño \\
\hline
\end{tabular} & \begin{tabular}{l} 
Cooke (1994) y McClymont y Jocumsen (2003) \\
\hline
\end{tabular}
\end{tabular}

Fuente: elaboración propia, a partir de la información de la empresa.

\section{METODOLOGÍA}

El objetivo principal del trabajo realizado es descubrir las variables clave o subyacentes en la aplicación del marketing de relaciones en las empresas colombianas. Para esto se elaboró una investigación descriptiva con el fin de determinar el estado actual y el comportamiento de las compañías colombianas en cuanto al nivel de adopción de las estrategias del marketing de relaciones. Esto se hizo en dos olas (tabla 1). La primera ola corresponde a 2003 y 2004 y la segunda a 2010 y 2011. El instrumento se aplicó por medio de la realización de entrevistas cara a cara. Estas contaron con la participación de dos entrevistadores.
El autor fue el entrevistador durante la primera ola. En la segunda se aplicó el instrumento —el mismo usado en la primera - a través de encuestas electrónicas. Este se diseñó en el software Gandia Quest. La población en la que se centró la investigación estuvo compuesta por empresas colombianas. Estas fueron definidas y clasificadas, considerando su tamaño, de acuerdo con lo indicado en la Ley de la República de Colombia No. 590 del 2000, que fue modificada por la Ley 905 de 2004 (artículo 2). Según esta Ley, y este criterio, una empresa con menos de diez trabajadores es considerada una microempresa, una con entre diez y cincuenta es una pequeña empresa, una con entre cincuenta y doscientos es una mediana 
empresa y una con más de doscientos es una gran empresa.

En la primera ola se contactaron empresas a través de estudiantes de posgrado en Marketing en Bogotá, ya que esta ciudad junto al departamento de Cundinamarca contienen el $43,4 \%$ del total de las compañías colombianas. Los cuestionarios se aplicaron presencialmente en aquellas universidades que ofrecían dichos programas. En la segunda ola se trabajó con una base de datos de 1793 empresas colombianas, que fue obtenida y actualizada a través de la compañía de telemarketing Meridiani Ltda.

En definitiva, se lograron 174 encuestas de las cuales 95 fueron diligenciadas a través de correo electrónico, a lo largo de tres envíos. Las 79 restantes correspondieron al envío físico del cuestionario a través de correo postal. Esto tras haber contactado previamente, por vía telefónica, a las personas encargadas de marketing en las compañías. Para estos fines, se contó con la colaboración de una empresa de telemarketing, que realizó 591 llamadas a la misma base de datos inicial, obteniendo una tasa de respuesta del 13,36\% (79 encuestas diligenciadas entre 591).

A continuación se presenta la tabla 2 , correspondiente al tamaño de la muestra obtenida:

Tabla 2. Distribución de la muestra

\begin{tabular}{l|c|c}
\hline \multicolumn{3}{c}{ Trabajo de campo } \\
\hline $\begin{array}{l}\text { Primera ola } \\
(2003-2004)\end{array}$ & 322 & $64,90 \%$ \\
\hline $\begin{array}{l}\text { Segunda ola } \\
(2010-2011)\end{array}$ & 174 & $35,10 \%$ \\
\hline Total & 496 & $100 \%$ \\
\hline
\end{tabular}

Fuente: elaboración propia.

Por último, el criterio de selección de las empresas a encuestar fue aleatorio, teniendo en cuenta que no hubieran respondido la encuesta o que no hubiesen manifestado el deseo de no ser contactados para el diligenciamiento de la misma. A continuación, se presenta el grupo de ítems valorados por las empresas colombianas en relación con el nivel de adopción del marketing de relaciones: 


\section{Tabla 3. Cuestionario aplicado}

Dentro de la estrategia de marketing de su empresa, se desea establecer en qué consiste dicha orientación. Se le presentará una serie de frases para que por favor las evalúe de la siguiente forma: primero, encontrará una escala que evalúa qué tan importante es para su empresa cada frase presentada. A continuación, encontrará una escala que evalúa el desempeño de su empresa en cada una de las frases presentadas. Así, usted evaluará a su empresa en importancia y desempeño con cada frase.

\begin{tabular}{|c|c|c|c|c|c|}
\hline \multirow{2}{*}{ IMPORTANCIA } & 1 & 2 & 3 & 4 & 5 \\
\cline { 2 - 6 } & Nada importante & Poco importante & Neutro & Importante & Muy importante \\
\cline { 2 - 6 }
\end{tabular}

DESEMPEÑO

\begin{tabular}{|c|c|c|c|c|}
\hline 1 & 2 & 3 & 4 & 5 \\
\hline $\begin{array}{c}\text { Pobre } \\
\text { desempeño }\end{array}$ & & & & Alto Desempeño \\
\hline
\end{tabular}

FRASE

a. Preocupación por crear y mantener contacto directo con los clientes.

b. Preocupación por crear y mantener contacto permanente con los proveedores.

c. Preocupación por crear y mantener contacto permanente con distribuidores e intermediarios.

d. Establecimiento de un diálogo continuo y sincero con los clientes.

e. Preocupación por agregar valor al cliente.

f. Preocupación por reducir los sacrificios hechos por el cliente.

g. Incremento constante de la satisfacción del cliente.

h. Preocupación por la calidad ofrecida y percibida por los clientes.

i. Esfuerzos concretos y permanentes por incrementar la fidelidad de los clientes.

j. Preocupación por generar en el cliente cada vez una mayor confianza en la marca respecto a los productos/servicios ofrecidos.

k. Preocupación por generar seguridad en el cliente con respecto a la marca.

1. Generación de credibilidad en la marca.

m. Orientación hacia la operación de venta.

n. Enfoque en las características intrínsecas de los productos/servicios.

o. Incremento del nivel de compromiso con los clientes.

p. Incremento de la relación con los clientes a través del tiempo.

q. Conocimiento del nivel de satisfacción de los empleados con la empresa.

r. Asignación de recursos para incrementar la satisfacción de los empleados con la empresa.

s. Generación de compromiso del cliente con la marca de la empresa.

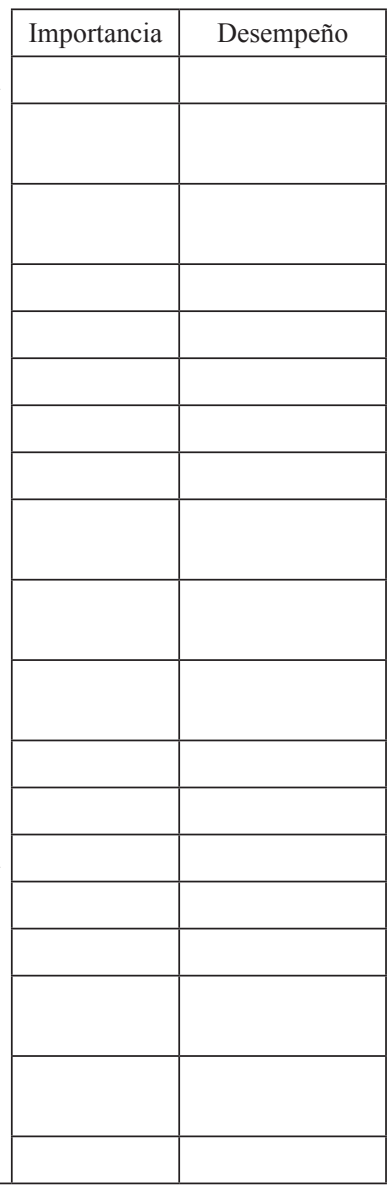

Fuente: elaboración propia 


\section{RESULTADOS}

Previo a la realización del análisis factorial de componentes principales planteado para esta investigación, se procedió a establecer la fiabilidad de las escalas a la luz del estadístico de fiabilidad Alfa de Cronbach (tabla 4).

Tabla 4. Estadísticos de Cronbach

\begin{tabular}{l|c|l|c}
\hline \multicolumn{2}{c|}{ Primera ola } & \multicolumn{2}{c}{ Segunda ola } \\
\hline \multicolumn{2}{c}{ Preguntas relativas a la importancia } \\
\hline Casos válidos & 322 & Casos válidos & 174 \\
\hline $\begin{array}{l}\text { Casos } \\
\text { excluidos }\end{array}$ & 0 & $\begin{array}{l}\text { Casos } \\
\text { excluidos }\end{array}$ & 0 \\
\hline $\begin{array}{l}\text { Elementos } \\
\text { (ítems) }\end{array}$ & 19 & $\begin{array}{l}\text { Elementos } \\
\text { (ítems) }\end{array}$ & 19 \\
\hline $\begin{array}{l}\text { Alfa de } \\
\text { Cronbach }\end{array}$ & 0,922 & $\begin{array}{l}\text { Alfa de } \\
\text { Cronbach }\end{array}$ & 0,905 \\
\hline
\end{tabular}

\begin{tabular}{l|c|l|c}
\hline \multicolumn{4}{c}{ Preguntas relativas al desempeño } \\
\hline Casos válidos & 322 & Casos válidos & 174 \\
\hline $\begin{array}{l}\text { Casos } \\
\text { excluidos }\end{array}$ & 0 & $\begin{array}{l}\text { Casos } \\
\text { excluidos }\end{array}$ & 0 \\
\hline $\begin{array}{l}\text { Elementos } \\
\text { (ítems) }\end{array}$ & 19 & $\begin{array}{l}\text { Elementos } \\
\text { (ítems) }\end{array}$ & 19 \\
\hline $\begin{array}{l}\text { Alfa de } \\
\text { Cronbach }\end{array}$ & 0,930 & $\begin{array}{l}\text { Alfa de } \\
\text { Cronbach }\end{array}$ & 0,923 \\
\hline
\end{tabular}

Fuente: elaboración propia.

Como se aprecia en la tabla 4 , todos los estadísticos de Cronbach para ambas olas y para ambas escalas, tanto los relativos a la importancia como los vinculados con el desempeño, se ubican por encima de 0,9 . De acuerdo con Pérez (2009), "valores por encima de 0,8 para el coeficiente Alfa indican consistencia interna muy aceptable para los elementos de la escala, $y$ valores por encima de 0,9 indican gran consistencia" (p. 215).

Acontinuación se presenta el análisis factorial de componentes principales, considerando un tamaño muestral mínimo de cinco observaciones por variable para cada una de las olas estudiadas (Hair et al., 1999) y para todos los ítems evaluados en relación con su importancia dentro de la estrategia de marketing de la empresa. Superando este valor mínimo sugerido de diecisiete observaciones por variable para la primera ola y nueve observaciones por variable para la segunda ola.

Como se puede apreciar en la tabla 5 , el test de Bartlett para ambas olas y por cada análisis de componentes es 0,0 . Por ende, se rechaza la hipótesis nula de la no correlación de las variables, es decir, aquella en la que todos los elementos son cero, exceptuando los de la diagonal, que son iguales a uno. 
Tabla 5. Análisis factorial para determinar el nivel de importancia que otorgan las empresas estudiadas a las variables del marketing de relaciones

\begin{tabular}{|c|c|c|c|}
\hline \multicolumn{2}{|l|}{ Primera ola } & \multicolumn{2}{|l|}{ Segunda ola } \\
\hline Prueba de esfericidad de Barlett & 0,912 & Prueba de esfericidad de Barlett & 0,868 \\
\hline Chi-cuadrado aproximado & 3545,738 & Chi-cuadrado aproximado & 1595,003 \\
\hline GL & 171 & GL & 171 \\
\hline Sig & 0.0 & Sig & 0.0 \\
\hline $\begin{array}{l}\text { Varianza total explicada en } \\
\text { cuatro factores }\end{array}$ & $66,497 \%$ & $\begin{array}{l}\text { Varianza total explicada en dos } \\
\text { factores }\end{array}$ & $65,568 \%$ \\
\hline $\begin{array}{l}\text { Variables con mayor carga } \\
\text { FACTOR } 1\end{array}$ & $23,933 \%$ & $\begin{array}{l}\text { Variables con mayor carga } \\
\text { FACTOR } 1\end{array}$ & $18,491 \%$ \\
\hline $\begin{array}{l}\text { - Crear y mantener el contacto } \\
\text { directo con los clientes }\end{array}$ & 0,773 & $\begin{array}{l}\text { - Generar seguridad en el cliente } \\
\text { con respecto a la marca }\end{array}$ & 0,806 \\
\hline \multirow{2}{*}{$\begin{array}{l}\text { - Incrementar constantemente la } \\
\text { satisfacción del cliente }\end{array}$} & \multirow{2}{*}{0,770} & - Generar credibilidad de marca & 0.798 \\
\hline & & \multirow{2}{*}{$\begin{array}{l}\text { - Generar cada vez mayor con- } \\
\text { fianza de marca con el cliente }\end{array}$} & \multirow{2}{*}{0,794} \\
\hline - Agregar valor al cliente & 0,727 & & \\
\hline \multirow{2}{*}{$\begin{array}{l}\text { - Establecer un diálogo continuo } \\
\text { y sincero con los clientes }\end{array}$} & \multirow[t]{2}{*}{0,709} & $\begin{array}{l}\text { Variables con mayor carga } \\
\text { FACTOR } 2\end{array}$ & $13,227 \%$ \\
\hline & & - Crear y mantener contacto & 0,754 \\
\hline - Generar credibilidad de marca & 0,700 & permanente con los proveedores & \\
\hline \multirow{2}{*}{$\begin{array}{l}\text { Variables con mayor carga } \\
\text { FACTOR } 2\end{array}$} & \multirow{2}{*}{$18,539 \%$} & $\begin{array}{l}\text { Preocupación por crear y } \\
\text { mantener contacto directo con } \\
\text { los clientes }\end{array}$ & 0,708 \\
\hline & & $\begin{array}{l}\text { Variables con mayor carga } \\
\text { FACTOR } 3\end{array}$ & $12,173 \%$ \\
\hline \multirow{2}{*}{$\begin{array}{l}\text { - Generar seguridad en el cliente } \\
\text { con respecto a la marca }\end{array}$} & \multirow[b]{2}{*}{0,867} & - Agregar valor al cliente & 0,866 \\
\hline & & $\begin{array}{l}\text { - Reducir los sacrificios del } \\
\text { cliente }\end{array}$ & 0,746 \\
\hline - Generar credibilidad de marca & 0,810 & $\begin{array}{l}\text { Variables con mayor carga } \\
\text { FACTOR } 4\end{array}$ & $11,050 \%$ \\
\hline \multirow[t]{2}{*}{$\begin{array}{l}\text { - Generar cada vez mayor con- } \\
\text { fianza de marca con el cliente }\end{array}$} & \multirow[t]{2}{*}{0,734} & $\begin{array}{l}\text { - Centrarse en las características } \\
\text { intrínsecas de los productos/ } \\
\text { servicios }\end{array}$ & 0,728 \\
\hline & & - Incrementar el nivel de & \\
\hline \multirow{2}{*}{$\begin{array}{l}\text { Variables con mayor carga } \\
\text { FACTOR } 3\end{array}$} & \multirow{2}{*}{$12,309 \%$} & $\begin{array}{l}\text { compromiso con los } \\
\text { clientes }\end{array}$ & 0,701 \\
\hline & & $\begin{array}{l}\text { Variables con mayor carga } \\
\text { FACTOR } 5\end{array}$ & $10,578 \%$ \\
\hline
\end{tabular}




\begin{tabular}{|c|c|c|c|}
\hline Primera ola & & Segunda ola & \\
\hline - Asignar recursos para incre- & 0,872 & $\begin{array}{l}\text { - Asignar recursos para incre- } \\
\text { mentar la satisfacción de los } \\
\text { empleados con la empresa }\end{array}$ & 0,826 \\
\hline $\begin{array}{l}\text { - Conocer el nivel de satis- } \\
\text { facción de los empleados } \\
\text { con la empresa }\end{array}$ & 0,797 & \multirow{4}{*}{$\begin{array}{l}\text { - Conocer el nivel de satisfacción } \\
\text { de los empleados con la empresa }\end{array}$} & \multirow{4}{*}{0,745} \\
\hline $\begin{array}{l}\text { Variables con mayor carga } \\
\text { FACTOR } 4\end{array}$ & $11,716 \%$ & & \\
\hline $\begin{array}{l}\text { - Crear y mantener contacto per- } \\
\text { manente con los proveedores }\end{array}$ & 0,788 & & \\
\hline $\begin{array}{l}\text { - Crear y mantener contacto } \\
\text { permanente con distribuidores } \\
\text { e intermediarios }\end{array}$ & 0,761 & & \\
\hline
\end{tabular}

Fuente: elaboración propia.

De acuerdo con lo indicado en la tabla 5, en la primera ola se descubren cuatro factores que, juntos, reúnen el $66,497 \%$ del total de la varianza explicada. El primer factor explica el $23,933 \%$. Este agrupa cinco ítems relativos al interés por los clientes, a saber: crear y mantener contacto directo con los clientes, incremento constante de la satisfacción del cliente, agregar valor al cliente, establecer un diálogo y generar credibilidad de marca. A este factor se le denominó 'importancia del cliente'.

El segundo factor, que explica el $18,539 \%$ de la varianza total, se compone de: la importancia por generar seguridad en el cliente con la marca, generar credibilidad y gene- rar confianza con la marca. A este se le denomino 'importancia de la generación de seguridad, credibilidad y confianza en el cliente'. El tercer factor, que explica el 12,309\% de la varianza total, se compone de: la importancia de asignar recursos para la satisfacción de los empleados y la importancia de conocer el nivel de satisfacción de los empleados. A este se le denominó 'importancia de los empleados'.

El cuarto factor explica el 11,716\% del total de la varianza. Se compone de: la importancia por el contacto con los proveedores y la importancia del contacto con distribuidores e intermediarios. A este se le denominó 'importancia del contacto con proveedores e intermediarios'. 
Por otra parte, en la segunda ola, se descubren cinco factores que en su conjunto explican el 65,568\% de la varianza total. El primer factor, que explica el $18,491 \%$ de la varianza, está compuesto por la importancia de generar en el cliente seguridad, confianza y credibilidad de marca. A este se le denominó 'importancia por generar seguridad, confianza y credibilidad de marca'.

El segundo factor, que explica el $13,227 \%$ de la varianza total, está compuesto por la importancia de estar en contacto con clientes y proveedores. A este se le denominó 'importancia del contacto con clientes y proveedores'. El tercer factor explica el $12,173 \%$ de la varianza total. Se compone de la importancia de agregar valor y reducir los sacrificios del cliente. A este se le denominó 'importancia por la satisfacción total del cliente'.

El cuarto factor, que explica el $11,050 \%$ de la varianza total, está compuesto por la importancia del producto/servicio base y la importancia del compromiso con los clientes. A este se le denominó 'importancia por la calidad y el compromiso'. El quinto factor, que explica el $10,578 \%$ de la varianza total, está compuesto por la importancia de asignar recursos para la satisfacción de los empleados y la de conocer el nivel de satisfacción de los empleados. A este se le denominó 'importancia de los empleados'.

La tabla 6 recoge el análisis factorial de componentes principales para todos los ítems evaluados en relación con el desempeño de la empresa.

Tabla 6. Análisis factorial para determinar la evaluación de desempeño que otorgan las empresas estudiadas a las variables del marketing de relaciones

\begin{tabular}{|l|c|l|c|}
\hline \multicolumn{2}{|c|}{ Primera ola } & \multicolumn{2}{c|}{ Segunda ola } \\
\hline Prueba de esfericidad de Barlett & 0,925 & Prueba de esfericidad de Barlett & 0,89 \\
\hline Chi-cuadrado aproximado & 3421,081 & Chi-cuadrado aproximado & 1815,362 \\
\hline GL & 171 & GL & 171 \\
\hline Sig & 0,0 & Sig & 0.0 \\
\hline $\begin{array}{l}\text { Varianza total explicada en } \\
\text { tres factores }\end{array}$ & $59,545 \%$ & $\begin{array}{l}\text { Varianza total explicada en } \\
\text { cuatro factores }\end{array}$ & $64,258 \%$ \\
\hline
\end{tabular}

Continúa 


\begin{tabular}{|c|c|c|c|}
\hline \multicolumn{2}{|l|}{ Primera ola } & \multicolumn{2}{|l|}{ Segunda ola } \\
\hline Variables con mayor carga & $45,396 \%$ & $\begin{array}{l}\text { Variables con mayor carga } \\
\text { FACTOR } 1\end{array}$ & 43,093 \\
\hline $\begin{array}{l}\text { Crear y mantener contacto } \\
\text { directo con los clientes }\end{array}$ & 0,762 & $\begin{array}{l}\text { Esfuerzos concretos y per- } \\
\text { manentes por incrementar la } \\
\text { fidelidad de los clientes }\end{array}$ & 0,669 \\
\hline $\begin{array}{l}\text { Establecer un diálogo continuo } \\
\text { y sincero con los clientes }\end{array}$ & 0,740 & $\begin{array}{l}\text { Variables con mayor carga } \\
\text { FACTOR } 2\end{array}$ & 8,349 \\
\hline $\begin{array}{l}\text { Incrementar constantemente la } \\
\text { satisfacción del cliente }\end{array}$ & 0,682 & Generar credibilidad de marca & 0,811 \\
\hline Agregar valor al cliente & 0,675 & $\begin{array}{l}\text { Preocupación por generar segu- } \\
\text { ridad en el cliente con respecto }\end{array}$ & 0,892 \\
\hline \multirow{2}{*}{$\begin{array}{l}\text { Variables con mayor carga } \\
\text { FACTOR } 2\end{array}$} & \multirow{2}{*}{$7,581 \%$} & a la marca & \\
\hline & & \multirow{3}{*}{$\begin{array}{l}\text { Preocupación por generar cada } \\
\text { vez mayor confianza de marca } \\
\text { en el cliente, con respecto a los } \\
\text { productos/servicios ofrecidos }\end{array}$} & \multirow{3}{*}{0,797} \\
\hline $\begin{array}{l}\text { Generar seguridad en el cliente } \\
\text { con respecto a la marca }\end{array}$ & 0,850 & & \\
\hline Generar credibilidad de marca & 0,794 & & \\
\hline \multirow{2}{*}{$\begin{array}{l}\text { Generar cada vez mayor con- } \\
\text { fianza de marca con el cliente }\end{array}$} & \multirow{2}{*}{0,763} & $\begin{array}{l}\text { Generar compromiso del cliente } \\
\text { con la marca de la empresa }\end{array}$ & 0,657 \\
\hline & & $\begin{array}{l}\text { Variables con mayor carga } \\
\text { FACTOR } 3\end{array}$ & 7,492 \\
\hline $\begin{array}{l}\text { Variables con mayor carga } \\
\text { FACTOR } 3\end{array}$ & $6,568 \%$ & $\begin{array}{l}\text { Preocupación por crear y man- } \\
\text { tener contacto directo con los }\end{array}$ & 0,793 \\
\hline \multirow{2}{*}{$\begin{array}{l}\text { Alta orientación hacia la } \\
\text { operación de venta }\end{array}$} & \multirow{2}{*}{0,659} & clientes & \\
\hline & & $\begin{array}{l}\text { Establece un diálogo continuo y } \\
\text { sincero con los clientes }\end{array}$ & 0,742 \\
\hline \multirow{4}{*}{$\begin{array}{l}\text { Preocupación por crear y man- } \\
\text { tener contacto con los provee- } \\
\text { dores }\end{array}$} & \multirow{4}{*}{0,655} & $\begin{array}{l}\text { Preocupación por crear y man- } \\
\text { tener contacto permanente con } \\
\text { los proveedores }\end{array}$ & 0,701 \\
\hline & & $\begin{array}{l}\text { Variables con mayor carga } \\
\text { FACTOR } 4\end{array}$ & 5,324 \\
\hline & & $\begin{array}{l}\text { Asignar recursos para incre- } \\
\text { mentar la satisfacción de los } \\
\text { empleados con la empresa }\end{array}$ & 0,854 \\
\hline & & $\begin{array}{l}\text { Conocer el nivel de satisfacción } \\
\text { de los empleados con la empresa }\end{array}$ & 0,815 \\
\hline
\end{tabular}

Fuente: elaboración propia. 
El factor número uno para las dos olas es el que explica el mayor porcentaje de la varianza retenida, siendo para la primera ola del $76 \%$ $(45,396 / 59,545)$, y para la segunda ola del $67 \%(43,093 / 64,258)$. Las variables relacionadas al factor uno en la primera ola son aquellas vinculadas con el relacionamiento del cliente. Por este motivo, a este factor se le denominó 'relación con el cliente'.

Por otra parte, en el primer factor de la segunda ola la variable que más correlación presenta es esfuerzos concretos y permanentes por incrementar la fidelidad de los clientes. A este se le denominó 'fidelidad de los clientes'. El segundo factor para las dos olas se denominó 'potencia de la marca' debido a que las variables relacionadas con este son aquellas que representan la fortaleza de la marca para la empresa. El tercer factor para la primera ola es un tanto ambiguo en su interpretación, sin embargo es más claro su comportamiento para la segunda ola. A este se le denominó 'contacto con clientes y proveedores'.

Finalmente, en la segunda ola es interesante ver surgir la importancia de los empleados como variable del marketing de relaciones. Por ese motivo, el cuarto factor para la segunda ola se denominó 'importancia de los empleados'.

\section{CONCLUSIONES}

El análisis factorial, como técnica de reducción de datos, permitió contrastar la validez del constructo propuesto y determinar cuáles son las variables influyentes en la adopción del marketing de relaciones para las empresas estudiadas. Haciendo referencia a esto es importante considerar lo indicado por García, Gil y Rodríguez (2000). Estos autores afirman que:

Un último aspecto a considerar en relación con el análisis factorial exploratorio como procedimiento para estimar la validez de constructo está estrechamente relacionado con la esencia misma de este tipo de análisis. No debe olvidarse que lo que nos ofrece el análisis factorial exploratorio son solo interpretaciones "post-hoc" de los factores y, por tanto, dichas interpretaciones no deben considerarse como evidencias de hipótesis formuladas a priori acerca de los constructos estudiados. Más bien deben entenderse como vías para aproximarse a la estructura interna de ciertas construcciones teóricas o como un modo de descubrirlas (p.18).

De acuerdo con lo hallado - $-\mathrm{y}$ presentado en las tablas 5 y 6 - se puede observar que no se presentan grandes diferencias en los resultados obtenidos ni en los periodos evaluados ni en el tipo de variables (relativas a 
la importancia y al de-sempeño). Por ejemplo, en la primera ola y para las variables referidas a la 'importancia' es fundamental para las empresas colombianas un enfoque de sus estrategias hacia el cliente. Este enfoque es seguido por la importancia del contacto con los empleados y con los intermediarios. Prima, de hecho, la importancia por el cliente.

En la segunda ola, el comportamiento encontrado es muy similar al de la primera, con casi las mismas variables vinculadas a la 'importancia'. En referencia a las relativas al 'desempeño' se presenta para ambas olas una coincidencia casi total en la orientación de la empresa hacia el cliente, en mayor proporción, seguida de una orientación hacia el fortalecimiento de la marca $y$, por último, del papel desempeñado por lo empleados. Acontinuación se presentan las variables clave del marketing de relaciones adoptadas por las empresas colombianas:

- Relación con el cliente.

- Fidelidad de los clientes.

- Potencia de la marca.

- Contacto con clientes y proveedores.

- Importancia de los empleados.

Al observar la esencia de las anteriores 'nuevas variables' descubiertas es posible identificar que las empresas colombianas asocian el concepto de marketing de relaciones, principalmente, con la relación con los clientes. Comprenden esto como un propósito fundamental de su orientación estratégica que privilegian sobre el hecho de tratar de establecer relaciones con otros actores más allá del entorno, los proveedores y canales de distribución y otros participantes en la relación. Esto sin entender y adoptar el concepto ampliado de marketing de relaciones que se incorpora en esta investigación.

El concepto del marketing de relaciones, a través del tiempo, no impactó a las empresas colombianas en su gestión estratégica. Se asumió en ellas este concepto como la relación a largo plazo con los clientes.

Se puede concluir que las empresas colombianas no tienen claro el concepto íntegro del marketing de relaciones. Se vislumbra que para estas el concepto de marketing de relaciones involucra débilmente algunos aspectos del cliente final y no los pertinentes a otros actores que influyen también en la relación. Se evidencia un claro desinterés en las empresas colombianas por adoptar el marketing de relaciones ajustado a la propuesta teórica planteada desde la escuela nórdica europea de marketing. 
Lo expuesto en este documento no significa necesariamente que las empresas colombianas estén siendo objeto de una gestión deficiente. Significa que para el empresario colombiano es más provechoso 'adoptar' las propuestas teóricas surgidas desde los países del primer mundo.

\section{REFERENCIAS}

Abramson, N. R., \& Ai, J. X. (1998). Practicing relationship marketing in Southeast Asia: Reducing uncertainty and improving performance. Management International Review, 38(1, Special issue), 113-143.

Aeron, H., Kumar, A., \& Moorthy, J (2012). Data mining framework forcustomer lifetimevalue-based segmentation. Database Marketing \& Customer Strategy Management, 19(1), 17-30.

Ali, A., Jolson, M. A., \& Darmon, R. Y. (1994). A model for optimizing the refund value in rebate promotions. Journal of Business Research, 29(3), 239-245.

Ballantyne, D. (1999, noviembre). Dialogue and knowledge generation: Two sides of the same coin in relationship marketing. Ponencia presentada en la 2nd WWW Conference on Relationship Marketing, Melbourne: Monash University.
Berry, L. L. (1995). Relationship marketing of services-growing interest, emerging perspectives. Journal of the Academy of Marketing Science, 23(4), 236-245.

Berry, L. L. (2002). Relationship marketing of services perspectives from 1983 and 2000. Journal of Relationship Marketing, 1(1), 59-77.

Bitner, M. J. (1995). Building service relationship: It's all about promises. Journal of the Academy of MarketingScience, 23(4), 246-251.

Bowen, J. T., \& Chen, S-L. (2001). The relationship between customer loyalty and customer satisfaction. International Journal of Contemporary Hospitality Management, 13(5), 213-217.

Butcher, K., Sparks, B., \& O'Callaghan, F. (2002). On the nature of customer-employee relationships. Marketing Intelligence \& Planning, 20(5), 297-306.

Cooke, S. (1994). Database marketing: Strategy or tactical tool? Marketing Intelligence \& Planning, 12(6), 4-7.

Cravens, D. W., \& Piercy, N. F. (1994). Relationship marketing and collaborativenetworks in service organizations. International Journal of Service Industry Management, 5(5), 39-53.

El-Ansary, A. I. (2005). Relationship marketing management: A 
school in the history of marketing thought. Journal of Relationship Marketing, 4(1), 43-56.

García, E., Gil, J., Rodríguez, G. (2000). Análisis factorial. Madrid: La Muralla.

Grönroos, C. (1990). Service managementandmarketing: Managing the moments of truth in service competition. Lexington: Lexington Books.

Grönroos, C. (1994). From marketing mix to relationship marketing: Towards a paradigm shift in marketing. Management Decision, 32(2), 4-20.

Grönroos, C. (2000). Service management and marketing: A customer relationship management approach (2nd ed.). Chichester: John Wiley \& Sons.

Gummesson, E. (1994). Making relationship marketing operational. International Journal of service industry management, 5(5), 5-20.

Gummesson, E. (1996). Relationship marketing and imaginary organizations: A synthesis. European Journal of Marketing, 30(2), 3141.

Hair, J., Anderson, R., Tatham, R., \& Black, W. (1999). Análisis multivariante ( $\left.5^{\mathrm{a}} \mathrm{ed}\right)$. Madrid: Prentice Hall.

Harker, J. (1999). Relationship marketing defined? An examination of current relationship marketing definitions. Marketing Intelligence \& Planning, 17(1), 13-20.

Kavali, S. G., Tzokas, N. X., \& Saren, M. J. (1999). Relationship marketing as an ethical approach: Philosophical and managerial considerations. ManagementDecision, 37(7), 573-581.

Linvol, H., \& Razzouk, N. (2006). From market share to customer share: Implications to marketing strategies. The Business Review, 5(1), 33-38.

McClymont, H., \& Jocumsen, G. (2003). How to implement marketing strategies using database approaches. Journal of Database Marketing \& Customer Strategy Management, 11(2), 135-148.

Morgan, R., \& Hunt, S. (1994).The commitment-trust theory of relationship marketing. Journal of Marketing, 58(3), 20-38.

Noble, C., \& Michael, M. (1999). Implementing marketing strategies: Developing and testing a managerial theory. Journal of Marketing, 63(4), 57-73.

Nwankwo, J. (1995). Developing a customerorientation.TheJournal of Consumer Marketing, 12(5), 5-15.

Pérez, C. (2009). Técnicas estadísticas multivariantes con SPSS. Madrid: Garceta Grupo Editorial. Piercy, N. (1998). Marketing implementation: The implications of 
marketing paradigm weakness forthestrategy execution process. Journal of the Academy of Marketing Science, 26(3), 222-236. Siems, F. (2010). Extending the life event cycle to relationship marketing: New implications for products and services. International Journal of Business Research, 10(2), 81-96.

Sin, L., Tse, A., Yau, O., Chow, R., Lee, J., \& Lau, L. (2005). Relationship marketing orientation: scale development and cross-cultural validation. Journal of Business Research, 58(2), 185-194.

Snow, C. (1997). Twenty-first-century organizations: Implications for a new marketing paradigm.
Journal of the Academy of Marketing Science, 25(1), 72-74.

Storbacka, K. (2000). Customer Profitability: Analysis and Design Issues. En J. N. Sheth \& A. Parvatiyar (Eds.), Handbook of Relationship Marketing (pp. 565586). Thousand Oaks: SAGE. Tadepalli, R., \& Ávila, R. (1999). Market orientation and the marketing strategy process. Journal of Marketing Theory and Practice, 7(2), 69-82.

Wrenn, B. (1997). The marketing orientation construct: Measurement and scaling issues. Journal of Marketing Theory and Practice, 5(3), 31-54. 